\title{
ON THE RESIDUE CLASS DISTRIBUTION OF THE NUMBER OF PRIME DIVISORS OF AN INTEGER
}

\author{
MICHAEL COONS AND SANDER R. DAHMEN
}

\begin{abstract}
Let $\Omega(n)$ denote the number of prime divisors of $n$ counting multiplicity. One can show that for any positive integer $m$ and all $j=0,1, \ldots, m-1$, we have

$$
\#\{n \leq x: \Omega(n) \equiv j(\bmod m)\}=\frac{x}{m}+o\left(x^{\alpha}\right),
$$

with $\alpha=1$. Building on work of Kubota and Yoshida, we show that for $m>2$ and any $j=0,1, \ldots, m-1$, the error term is not $o\left(x^{\alpha}\right)$ for any $\alpha<1$.
\end{abstract}

\section{$\S 1$. Introduction}

The Liouville function, denoted $\lambda(n)$, is defined by $\lambda(n):=(-1)^{\Omega(n)}$, where $\Omega(n)$ is the number of prime divisors of $n$ counting multiplicity. The Liouville function is closely connected to the Riemann zeta function and hence to many results and conjectures in prime number theory. Recall from [5, pp. 617-621] that for $\Re s>1$, we have

$$
\sum_{n \geq 1} \frac{\lambda(n)}{n^{s}}=\frac{\zeta(2 s)}{\zeta(s)}
$$

so that $\zeta(s) \neq 0$ for $\Re s \geq \vartheta$, provided that $\sum_{n \leq x} \lambda(n)=o\left(x^{\vartheta}\right)$. The prime number theorem allows the value $\vartheta=1$, so that for $j=0,1$, we have

$$
\#\{n \leq x: \Omega(n) \equiv j(\bmod 2)\} \sim \frac{x}{2} .
$$

If the Riemann hypothesis holds, we even have, for $j=0,1$ and every $\alpha>$ $1 / 2$,

$$
\#\{n \leq x: \Omega(n) \equiv j(\bmod 2)\}=\frac{x}{2}+o\left(x^{\alpha}\right)
$$

Received February 6, 2010. Revised May 19, 2010. Accepted May 23, 2010.

2010 Mathematics Subject Classification. Primary 11N37, 11N60; Secondary 11N25, $11 \mathrm{M} 41$.

Coons's work supported by a Fields-Ontario Fellowship. Dahmen's work supported by the Natural Sciences and Engineering Research Council of Canada.

(C) 2011 by The Editorial Board of the Nagoya Mathematical Journal 
Kubota and Yoshida [4] investigated whether similar asymptotic properties could hold in general for the functions

$$
N_{m, j}(x):=\#\{n \leq x: \Omega(n) \equiv j(\bmod m)\}, \quad m \in \mathbb{Z}_{>0}, j=0,1, \ldots, m-1
$$

To this end, they introduced and studied generalizations of the Liouville function.

The question of whether for all $m \in \mathbb{Z}_{>0}$ and $j=0,1, \ldots, m-1$, we have

$$
N_{m, j}(x)=\frac{x}{m}+o\left(x^{\alpha}\right)
$$

with $\alpha=1$ left open by Kubota and Yoshida [4], but it turns out that this follows from a result of Rivat, Sárközy, and Stewart [6]. In Section 2, we show that this also follows very quickly from a result of Hall [3] on the mean values of multiplicative functions.

As for the question of whether (1) can hold with $\alpha<1$ if $m>2$, Kubota and Yoshida obtained the following surprising result.

Theorem 1 ([4, Theorem 4]). Let $m \in \mathbb{Z}_{>2}$, and let $\alpha<1$. Then for at least one $j=0,1, \ldots, m-1$, we have that (1) does not hold.

This is in striking contrast to the expected result for $m=2$. The result of Kubota and Yoshida still leaves open the possibility that, for some $m>2$ and some $j=0,1, \ldots, m-1$, equation (1) holds with some $\alpha<1$. Our main result is that this is impossible.

Theorem 2. Let $m \in \mathbb{Z}_{>2}$, and let $\alpha<1$. Then for all $j=0,1, \ldots, m-1$, equation (1) does not hold.

A proof, building on the work of Kubota and Yoshida [4], is given in Section 3.

\section{$\S 2$. Generalizations of the Liouville function}

Let $m \in \mathbb{Z}_{>0}$, and let $\zeta_{m}:=e^{2 \pi i / m}$ be a primitive $m$ th root of unity. As a generalization of Liouville's function, define for $k=0,1, \ldots, m-1$ the function

$$
\lambda_{m, k}(n):=\zeta_{m}^{k \Omega(n)} .
$$

The functions $\lambda_{m, k}(n)$ were introduced by Kubota and Yoshida [4] to study the asymptotics of $N_{m, j}(x)$ for $m>2$. To investigate the properties of 
$N_{m, j}(x)$, it is natural to look at the partial sums

$$
S_{m, k}(x):=\sum_{n \leq x} \lambda_{m, k}(n) .
$$

First of all, there is a simple but very useful linear relationship between $S_{m, k}(x)$ and $N_{m, j}(x)$. For $k=0,1, \ldots, m-1$, we have

$$
S_{m, k}(x)=\sum_{n \leq x} \zeta_{m}^{k \Omega(n)}=\sum_{j=0}^{m-1} \sum_{\substack{n \leq x \\ \Omega(n) \equiv j(\bmod m)}} \zeta_{m}^{k \Omega(n)}=\sum_{j=0}^{m-1} \zeta_{m}^{k j} N_{m, j}(x) .
$$

Conversely, for $j=0,1, \ldots, m-1$, we have

$$
\begin{aligned}
N_{m, j}(x) & =\sum_{\substack{n \leq x \\
\Omega(n) \equiv j(\bmod m)}} 1=\sum_{n \leq x} \frac{1}{m} \sum_{k=0}^{m-1} \zeta_{m}^{k(\Omega(n)-j)} \\
& =\frac{1}{m} \sum_{k=0}^{m-1} \zeta_{m}^{-j k} S_{m, k}(x) .
\end{aligned}
$$

Second, since $\lambda_{m, k}(n)$ is a multiplicative function with values in the unit disk, we can apply the following theorem of Hall [3] to give an asymptotic bound of $S_{m, k}(x)$.

Theorem 3 (see [3]). Let $D$ be a convex subset of the closed unit disk in $\mathbb{C}$ containing zero with perimeter $L(D)$. If $f: \mathbb{Z}_{>0} \rightarrow \mathbb{C}$ is a multiplicative function with $|f(n)| \leq 1$ for all $n \in \mathbb{Z}_{>0}$ and $f(p) \in D$ for all primes $p$, then

$$
\frac{1}{x}\left|\sum_{n \leq x} f(n)\right| \ll \exp \left(-\frac{1}{2}\left(1-\frac{L(D)}{2 \pi}\right) \sum_{p \leq x} \frac{1-\Re f(p)}{p}\right) .
$$

Lemma 4. For every $m \in \mathbb{Z}_{>0}$ there exists an $A>0$ such that for all $k=1,2, \ldots, m-1$, we have

$$
\left|S_{m, k}(x)\right| \ll \frac{x}{\log ^{A} x} .
$$

Proof. Set $D$ equal to the convex hull of the $m$ th roots of unity, and set $f(n)=\lambda_{m, k}(n)$. Because $D$ is a convex subset strictly contained in the closed unit disk of $\mathbb{C}$, we have $L(D)<2 \pi$. This gives

$$
c:=\frac{1}{2}\left(1-\frac{L(D)}{2 \pi}\right)>0 \text {. }
$$


Applying Theorem 3 yields

$$
\frac{1}{x}\left|\sum_{n \leq x} \lambda_{m, k}(n)\right| \ll \exp \left(-c \sum_{p \leq x} \frac{1-\Re \lambda_{m, k}(p)}{p}\right)=\exp \left(-c\left(1-\Re \zeta_{m}^{k}\right) \sum_{p \leq x} \frac{1}{p}\right) .
$$

Since $\sum_{p \leq x} p^{-1}=\log \log x+O(1)$, this quantity is

$$
\ll \exp \left(-c\left(1-\Re \zeta_{m}^{k}\right) \log \log x\right)=\left(\frac{1}{\log x}\right)^{c\left(1-\Re \zeta_{m}^{k}\right)}
$$

Noting that $0<k<m$, we have $c\left(1-\Re \zeta_{m}^{k}\right)>0$. Set $A:=\min _{0<k<m}\{c(1-$ $\left.\left.\Re \zeta_{m}^{k}\right)\right\}$. Then $A>0$, and we obtain

$$
\left|\sum_{n \leq x} \lambda_{m, k}(n)\right| \ll \frac{x}{\log ^{A} x} .
$$

As in the work of Rivat, Sárközy, and Stewart [6], this bound for the partial sums $S_{m, k}(x)$ immediately leads to an asymptotics result for the counting functions $N_{m, j}(x)$.

Corollary 5. Let $m \in \mathbb{Z}_{>0}$. There exists an $A>0$ (depending on $m$ ) such that for all $j=0,1, \ldots, m-1$, we have

$$
N_{m, j}(x)=\frac{x}{m}+O\left(\frac{x}{\log ^{A} x}\right) .
$$

In particular, for all $j=0,1, \ldots, m-1$, we have that (1) holds with $\alpha=1$.

Proof. From (3) we immediately get

$$
N_{m, j}(x)=\frac{1}{m} S_{m, 0}(x)+\frac{1}{m} \sum_{k=1}^{m-1} \zeta_{m}^{-j k} S_{m, k}(x) .
$$

The first term of the right-hand side of (5) is

$$
\frac{1}{m} S_{m, 0}(x)=\frac{1}{m} \sum_{n \leq x} 1=\frac{x}{m}+O(1) .
$$

Applying the triangle inequality and Lemma 4, we get that the absolute value of the second term of the right-hand side of (5) is

$$
\left|\frac{1}{m} \sum_{k=1}^{m-1} \zeta_{m}^{-j k} S_{m, k}(x)\right| \leq \frac{1}{m} \sum_{k=1}^{m-1}\left|S_{m, k}(x)\right| \ll \frac{x}{\log ^{A} x}
$$

for some $A>0$. This gives us our desired result. 
The constant $A$ in Corollary 5 can easily be made explicit, but it is not the purpose of this paper to determine a good value for $A$. Readers interested in the constant $A$ may wish to consult [6].

\section{$\S 3$. Lower bounds for the error terms}

Let $m \in \mathbb{Z}_{>0}$, and let $j=0,1, \ldots, m-1$. We introduce the error term

$$
R_{m, j}(x):=N_{m, j}(x)-\frac{x}{m}
$$

Our main result, Theorem 2, obviously translates as follows.

Theorem 6. Let $m \in \mathbb{Z}_{>2}$, and let $\alpha<1$. None of $R_{m, 0}, R_{m, 1}, \ldots, R_{m, m-1}$ are $o\left(x^{\alpha}\right)$.

To prove Theorem 6, keeping with [4], we use the following results.

Lemma 7. Let $\left\{a_{n}\right\}_{n \in \mathbb{Z}_{>0}}$ be a sequence of complex numbers, and let $\alpha>0$. If the partial sums satisfy $\sum_{n \leq x} a_{n}=o\left(x^{\alpha}\right)$, then the Dirichlet series $\sum_{n \geq 1} a_{n} n^{-s}$ converges for $\Re s>\alpha$ to a holomorphic (single-valued) function.

Proof. This follows directly from Perron's formula (see [1, p. 243, Lemma 4]).

For $\Re s>1$, denote

$$
L_{m, k}(s):=\sum_{n \geq 1} \frac{\lambda_{m, k}(n)}{n^{s}}
$$

Kubota and Yoshida [4] introduced the function $L_{m, k}(s)$ and gave a multivalued analytic continuation of $L_{m, 1}(s)$ to the region $\Re s>1 / 2$; their proof easily generalizes to give the result for all $k=1,2, \ldots, m-1$; thus, we attribute to them the generalization as well.

Theorem 8 (see [4]). Let $m \in \mathbb{Z}_{>2}$, and let $k=1,2, \ldots, m-1$. The Dirichlet series $L_{m, k}(s)$ can be analytically continued to a multivalued function on $\Re s>1 / 2$ given by the product $\zeta(s)^{\zeta_{m}^{k}} G_{m, k}(s)$, where $G_{m, k}(s)$ is a holomorphic function for $\Re s>1 / 2$. In particular, if $k \neq m / 2$, then for any $\alpha<1$, the Dirichlet series $L_{m, k}(s)$ does not converge for all $s$ with $\Re s>\alpha$. 
Proof. The first part follows from (the proof of) [4, Theorem 1]. Note that $\zeta_{m}^{k}$ is not rational for $k \neq m / 2$. Since $\zeta(s)$ has a pole at $s=1$, this means that no branch of $\zeta(s)^{\zeta_{m}^{k}}$ is holomorphic in a neighborhood of $s=1$.

REMARK 9. Using these results, we can quickly obtain that if $m>2$, at least two of the error terms are not $o\left(x^{\alpha}\right)$ for any $\alpha<1$. For $k=1,2, \ldots$, $m-1$, using (2), we have

$$
S_{m, k}(x)=\sum_{j=0}^{m-1} \zeta_{m}^{j k} R_{m, j}(x) .
$$

By Lemma 7 and Theorem $8, S_{m, 1}(x)$ is not $o\left(x^{\alpha}\right)$ for any $\alpha<1$, so that at least one of the error terms $R_{m, j}(x)$ is not $o\left(x^{\alpha}\right)$, which is the result of Kubota and Yoshida [4, Theorem 1]. From (2) with $k=0$, we obtain

$$
\sum_{j=0}^{m-1} R_{m, j}(x)=S_{m, 0}(x)-x=-\{x\},
$$

where $\{x\}$ denotes the fractional part of $x$. This shows that it is impossible that all but one of the error terms $R_{m, j}(x)$ are $o\left(x^{\alpha}\right)$ for an $\alpha<1$.

Let $m>2$, and let $j=0,1, \ldots, m-1$. From (3) we get

$$
R_{m, j}(x)=\frac{1}{m} \sum_{k=1}^{m-1} \zeta_{m}^{-j k} S_{m, k}(x)-\frac{\{x\}}{m} .
$$

In light of Lemma 7, to obtain that $R_{m, j}(x)$ is not $o\left(x^{\alpha}\right)$ for any $\alpha<1$, it suffices to show that the generating function of $R_{m, j}(x)+\{x\} / m$, which is

$$
\frac{1}{m} \sum_{k=1}^{m-1} \zeta_{m}^{-j k} L_{m, k}(s)
$$

cannot be analytically continued to a holomorphic (single-valued) function in the half-plane $\Re s>\alpha$.

We now proceed with the proof of Theorem 6 .

Proof of Theorem 6 . Let $1 / 2<\alpha<1$, and let $c_{1}, c_{2}, \ldots, c_{m-1} \in \mathbb{C}^{*}$. We will prove that the linear combination

$$
f(s):=\sum_{k=1}^{m-1} c_{k} L_{m, k}(s)
$$


cannot be analytically continued to a holomorphic (single-valued) function in the half-plane $\Re s>\alpha$. Suppose, to the contrary, that it can, and assume for now that $L_{m, 1}(s), L_{m, 2}(s), \ldots, L_{m, m-1}(s)$ are linearly independent over $\mathbb{C}$, which will be shown later. Let $C$ denote a smooth path in the half-plane $\Re s>\alpha$, starting and ending in an $s_{0}$ with $\Re s_{0}>1$, winding around $s=1$ once in the positive direction and not winding around (and not passing) any zeros of $\zeta(s)$. (One way to obtain rigorous statements below is to consider all linear combinations of $L_{m, k}(s)$ and analytic continuations along $C$ thereof as single-valued holomorphic functions in the half-plane $\Re s>1$.) By Theorem 8, as pointed out in [4, Remark 1], the analytic continuation of $L_{m, k}(s)$ along $C$ gives us $\exp \left(-2 \pi i \zeta_{m}^{k}\right) L_{m, k}(s)$. From the holomorphicity assumption on $f(s)$, it follows that the analytic continuation of $f(s)$ along $C$ is $f(s)$ itself. So we have

$$
\sum_{k=1}^{m-1} c_{k} L_{m, k}(s)=\sum_{k=1}^{m-1} c_{k} \exp \left(-2 \pi i \zeta_{m}^{k}\right) L_{m, k}(s),
$$

and from the linear independence over $\mathbb{C}$ of the functions $L_{m, k}(s)$, we obtain that $\exp \left(-2 \pi i \zeta_{m}^{k}\right)=1$ for $k=1,2, \ldots, m-1$. This means that $\zeta_{m}^{k} \in \mathbb{Z}$ for $k=1,2, \ldots, m-1$, a contradiction if $m>2$.

We are left with proving that $L_{m, 1}(s), L_{m, 2}(s), \ldots, L_{m, m-1}(s)$ are linearly independent over $\mathbb{C}$. By the uniqueness of Dirichlet series (see, e.g., [1, Theorem 11.3]), this would follow from the linear independence over $\mathbb{C}$ of the functions $\lambda_{m, k}(n)=\zeta_{m}^{k \Omega(n)}$ for $k=1,2, \ldots, m-1$. To prove the latter, suppose that for some $d_{1}, d_{2}, \ldots, d_{m-1} \in \mathbb{C}$, we have that $\sum_{k=1}^{m-1} d_{k} \zeta_{m}^{k \Omega(n)}=0$ for all $n \in \mathbb{Z}_{>0}$. Then, in particular, $\sum_{k=1}^{m-1} d_{k}\left(\zeta_{m}^{k}\right)^{i}=0$ for $i=0,1, \ldots$, $m-2$. This defines a system of linear equations in the $d_{k}$ with matrix $M$ of Vandermonde type. The values $\zeta_{m}^{k}$ for $k=1,2, \ldots, m-1$ are all distinct, so $\operatorname{det} M \neq 0$. Therefore, $d_{1}, d_{2}, \ldots, d_{m-1}$ must all be zero; that is, $\lambda_{m, 1}(n), \lambda_{m, 2}(n), \ldots, \lambda_{m, m-1}(n)$ are linearly independent over $\mathbb{C}$. This completes the proof.

REMARK 10. In the spirit of prime number races, it seems fitting that further study should be taken to investigate the sign changes of $N_{m, j}(x)-$ $N_{m, j^{\prime}}(x)$ for $j \neq j^{\prime}$. For the case $m=2$, some such investigations have been undertaken (see [2] and the references therein).

Acknowledgment. The authors thank one of our referees for providing helpful comments, and in particular for suggesting a simplification of the proof of Theorem 6 . 


\title{
REFERENCES
}

[1] T. M. Apostol, Introduction to Analytic Number Theory, Undergrad. Texts Math., Springer, New York, 1976.

[2] P. Borwein, R. Ferguson, and M. J. Mossinghoff, Sign changes in sums of the Liouville function, Math. Comp. 77 (2008), 1681-1694.

[3] R. R. Hall, A sharp inequality of Halász type for the mean value of a multiplicative arithmetic function, Mathematika 42 (1995), 144-157.

[4] T. Kubota and M. Yoshida, A note on the congruent distribution of the number of prime factors of natural numbers, Nagoya Math. J. 163 (2001), 1-11.

[5] E. Landau, Handbuch der Lehre von der Verteilung der Primzahlen, 2 Bände, 2nd ed., with an appendix by P. T. Bateman, Chelsea, New York, 1953.

[6] J. Rivat, A. Sárközy, and C. L. Stewart, Congruence properties of the $\Omega$-function on sumsets, Illinois J. Math. 43 (1999), 1-18.

\author{
Michael Coons \\ University of Waterloo \\ Department of Pure Mathematics \\ Waterloo, Ontario \\ N2L $3 G 1$ \\ Canada \\ mcoons@math . uwaterloo.ca \\ Sander R. Dahmen \\ Mathematisch Instituut \\ Universiteit Utrecht \\ P.O. Box 80010 \\ 3508 TA Utrecht \\ The Netherlands \\ s.r.dahmen@uu.nl
}

